\title{
Translation and Validity of the Malay Version of the Five-Factor Nonverbal Personality Questionnaire (FFNPQ)
}

\author{
NF Ab Rashid ${ }^{a}$, WN Arifin ${ }^{a}$, YC Kueh ${ }^{a}$ and N Yusoff ${ }^{b}$ \\ ${ }^{a}$ Unit of Biostatistics and Research Methodology, School of Medical Sciences, Health Campus, Universiti Sains \\ Malaysia \\ ${ }^{b}$ Department of Neurosciences, School of Medical Sciences, Health Campus, Universiti Sains Malaysia
}

\section{ABSTRACT}

Introduction: The Five-Factor Nonverbal Personality Questionnaire (FF-NPQ) is a non-verbal personality inventory consisting of illustrations of different social situations, designed to measure five broad factors underlying the Big-Five model of personality. This study aimed to prove the validity of the FF-NPQ in Malaysia. Methods: The FF-NPQ instruction and the answer option were translated to the Malay language, which is the main language spoken in Malaysia. A total of 153 university students aged 18-30 years from Universiti Sains Malaysia were involved in this study. Confirmatory factor analysis (CFA), composite reliability and intraclass correlation (ICC) were applied to show evidence of validity by internal structure. Results: CFA indicated that only 26 out of 60 items remained in the final model while maintaining the five personality factors. Each factor had a good composite reliability value of $0.68-0.77$. The ICC values from the test-retest analysis ranged from 0.65 to 0.75 . Conclusion: Overall, the shortened Malay Version of the FF -NPQ (five-factor, 26 items) exhibited acceptable evidence of validity in Malaysia.

KEYWORDS: Big Five personality, FF-NPQ Malay Version, non-verbal questionnaire, validity INTRODUCTION

Personality can be defined as the unique pattern of thoughts, feelings and behaviours of an individual. ${ }^{1}$ Different individual may have different personalities that may change over time and across situations. ${ }^{2}$

Big Five is a well-known theory of five types of personality dimensions. The dimensions are Extraversion-sociality and outgoings of a person, Agreeableness-prosocial and communal orientation toward others with antagonism, Conscientiousness socially prescribed impulse control that facilitates task-directed and goal-directed behaviour, Neuroticism-emotional stability and temperedness with negative emotionality and Openness to experience-breadth, depth, originality

Corresponding Author:

Dr. Nasir Yusoff,

Department of Neurosciences,

School of Medical Sciences,

Health Campus Universiti Sains Malaysia,

16150 Kubang Kerian, Kelantan, Malaysia.

Tel No: 60-09-7676300/017-9788554

Fax No: 60-09-7676301

E-mail: nasiryusoff2310@gmail.com and complexity of the mental and experiential life..$^{3-}$ ${ }^{4}$ The Big Five frameworks were the most widely used by researchers in modelling the personality. ${ }^{5}$

The Five-Factor Nonverbal Personality Questionnaire (FF-NPQ) was designed to measure five broad factors underlying Five-Factor Model (FFM) of personality structure. Items in FF-NPQ was a subset of Nonverbal Personality Questionnaire (NPQ) items with 7-point scale where 1 indicate 'extremely unlikely' and 7 indicate 'extremely likely', while 4 indicate 'neither likely nor unlikely' ${ }^{6}$

In a study involving 701 university students from seven cultures to examine the psychometric

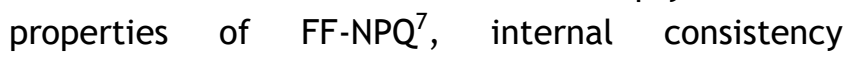
reliability, Cronbach's alpha coefficient ranged from 0.64 to 0.77 , with an average of 0.72 . The seven cultures included were Canada, England, Finland, Germany, Norway, Poland and Russia. Finland showed the lowest reliability over the five scales with mean of .66 , while highest reliability obtained by English data with a mean of 0.79 . The mean scale reliability for Canada, Germany, Norway, Poland and Russia were 0.75, 0.69, 0.71, 0.68 and 
0.72 , respectively. In the same study, convergent and discriminant correlations were also determined between FF-NPQ and Personality Research Form (PRF) Big Five scales. The convergent correlations were in range of $r=0.35$ (Neuroticism) to $r=0.54$ (Extraversion), with an average of $r=0.48$. The highest discriminant correlation was $r=0.26$. In term of convergent validity by country, the mean across five scales, were between 0.40 (Finland) and 0.55 (Norway and England).

Items in FF-NPQ are different from other personality inventories because they are presented in the form of illustrations of different social situations instead of verbal statements. Nonverbal measure has some advantages compared to verbal measures for studies across culture since it does not need to translate the item measures. ${ }^{7}$ It is also very useful when the study involves people having difficulty in reading and understanding language due to dyslexia or aging. ${ }^{6}$ However, some illustrations may not be relevant for different cultures because there might be differences in geographic, political environment and economic. ${ }^{7}$ Thus, this study was aimed to provide evidence of validity of Malay Version of FFNPQ in Malaysia.

\section{MATERIALS AND METHODS}

\section{Study Design and Participants}

This cross-sectional study involved 153 students (aged 18-30 years old) from Health Campus, Universiti Sains Malaysia (USM). Two approaches were used to justify the required sample size, as based on the sample size determination for Confirmatory Factor Analysis (CFA). First, the sample size was calculated by computing power and minimum sample size for RMSEA. ${ }^{8}$ Alpha level was set to 0.05 with power of study of 0.80 . The perfect fit RMSEA for null RMSEA (0.00) and exact fit RMSEA for alternative RMSEA (0.05) were fixed, with the calculation of the degree of freedom as below ${ }^{9}$ :

$$
\mathrm{df}=\mathrm{b}-\mathrm{a}
$$

From the equation,

$$
b=\frac{p(p+1)}{2}
$$

Where,

$b=$ number of elements of the input matrix (number of knowns)

$\mathrm{p}=$ number of the indicators included (number of items)
Therefore,

$$
b=\frac{60(60+1)}{2}=1830
$$

$a=$ number of freely estimated parameters (number of unknowns)

Where a consists of:

- Number of factor loadings $=5$ factors $\times(12$ items 1) $=55$

- Number of error variance $=5$ factors $\times 12$ items $=$ 60

- Number of factor variance $=5$

- Number of factor covariance $=10$

Therefore, $a=55+60+5+10=130$.

Thus, $\mathrm{df}=1830-130=1700$.

As referred to Brown', it was suggested that, appropriate sample size for the 1700 degree of freedom to achieve the RMSEA of 0.05 is about 37 respondents.

In the second approach, simulation study was used to determine the sample size through the CFA. ${ }^{10}$ Based on simulation study, the sample size is fixed at $\mathrm{n}=150$ when the expected constructs are seven or less and items commonality is less than 0.5 and no under identified constructs.

From these two approaches, the largest number of appropriate sample size was considered as the number of samples for CFA, which was 150 .

Stratified random sampling was applied in selecting the participants in which the name list of respondents was obtained from the Academic Office. Respondents with a lifetime history of a major medical disorder, uncorrected visual acuity, history of affective disorder and using psychiatric medication, as well as those who were not fluent in Malay language, were excluded from the study. The participants were then selected randomly from the list according to the size of the stratum. In the next stage, forty-four students were then randomly selected from the 153 students for the test-retest reliability analysis. The study protocol was approved by the Human Ethics Committee of USM (JEPeM USM Code: USM/JEPeM/15040127) and consent forms were distributed to each respondent before the data collection.

FF-NPQ 
A non-verbal measure of the FF-NPQ consists of 60 illustrations representing five personality factors (i.e. Extraversion, Agreeableness, Conscientiousness, Neuroticism and Openness, to experience) with 12 illustrations each, which is useful to be used among those with difficulty in reading and understanding language. Each illustration is rated on seven likert scales, ranging from one ('extremely unlikely') to seven ('extremely likely'). ${ }^{6}$ An instruction of the FF-NPQ and the answer option were translated to the Malay language as it is the main language spoken in Malaysia. Forward and backward translation methods were applied. In this method, four independent translators were involved during translation process; two translators for forward translation and two translators for backward translation $^{11-12}$ Translators were independent individuals who could speak and understand both languages. During forward translation process, the instruction was translated from English to Malay, while during backward translation process the instruction was translated back from Malay to English.

The translated FF-NPQ instruction versions from forward translator-1 and forward translator-2 were compared. Each word and sentences were compared to maintain the original meaning of the words and sentences. There was no difference in meaning of the sentences and words found from the versions. The versions were then harmonized to produce the forward-translated version of Malay FF$N P Q$ instruction.

The forward-translated version of Malay FF-NPQ instruction was then given to backward translator-1 and backward translator-2 to translate it back to English. The backward-translated versions from each translator were compared to the original English version. Problematic words and sentences were identified whenever discrepancies were found between the backward-translated version and the original English version. Only one word, 'likely', was found to be different between backwardtranslator 1 and backward-translator-2. Backtranslator-1 translated as 'possibility', while backtranslator-2 translated as 'probability'. However, the meaning of the original word was maintained. A preliminary Malay FF-NPQ instruction was produced following the process.

FF-NPQ with the preliminary FF-NPQ instruction was further investigated by conducting detailed cognitive debriefing sessions involving five respondents. They were asked by the researcher if there any words or phrases that were confusing and how they understood the instruction. They were asked to give comments and suggestions regarding the instruction. Following the cognitive debriefing process, FF-NPQ was pre-tested among 20 respondents. These respondents were asked to give their comments about the clarity and the arrangement of the instruction. In addition, the time takes for them to complete FF-NPQ was recorded to estimate the time taken to complete the questionnaire for real samples. Comments from the respondents at these two stages were considered to revise the preliminary Malay FF-NPQ instruction to produce the finalized Malay FF-NPQ instruction for use in the validation study.

\section{Data Entry and Statistical Software}

MS Excel 2007 was used for the data entry and the determination of cutoff values for each factor score. The statistical program of $R$ version 3.2.3 was used for the preliminary data screening, confirmatory factor analysis (CFA) and composite reliability. Testretest reliability analysis was conducted by using IBM SPSS version 22.

\section{Internal Structure}

CFA using the robust maximum likelihood estimator (MLR) was applied to determine the construct validity of the FF-NPQ by internal structure evidence. MLR was used because the data did not follow a multivariate normal distribution required by the MLR.

The assessment of multivariate normality was conducted using Mardia's test and plot of chisquared versus squared Mahalanobis distance. ${ }^{13-14}$ The assumption of multivariate normality is fulfilled when the critical ratio of multivariate kurtosis is less than 1.96 and the $p$-value is more than $0.05^{13-14}$, and when the plot shows that the data points form an approximately straight line. ${ }^{15}$

The CFA model was evaluated for model fit, the magnitude of factor loading and the correlation between factors. ${ }^{16}$ Four fit indices were chosen to evaluate the model fit: comparative fit index (CFI), Tucker-Lewis index (TLI), root mean square error of 
approximation (RMSEA) and standardised root mean square residual (SRMR). ${ }^{17}$ It is suggested that the characteristics of fit indices that demonstrate goodness-of-fit as CFI $\geq 0.95$, TLI $\geq 0.95$, RMSEA $\leq$ 0.06 and SRMR $\leq 0.08 .^{18}$ Factor loadings were considered satisfactory when the values were higher than 0.40 . $^{19}$ Thus, all items with a factor loading of less than 0.40 were removed from the model. Multicollinearity exists when the correlation among latent variables is more than 0.85 , which indicates poor discrimination among the factors. ${ }^{17}$

Modifications of the model were made on the basis of low factor loadings, high suggested modification indices (MI) and high standardised residual values with the fit indices. The recommended cutoff value for the standardised residual is $2.58^{17}$, which corresponds to the alpha of 0.01 of a $z$-distribution. It is proposed that standardised residuals with an absolute value between 2.5 and 4.0 require attention. ${ }^{20}$ The $\mathrm{Ml}$ with a value of 10 or greater suggests that the fit of the model can be improved. ${ }^{20}$ Decreasing Akaike Information Criteria (AIC) and Bayesian Information Criterion (BIC) values indicate improvements of the model fit. ${ }^{17}$ The model with the smallest AIC and BIC values is considered to have a good fit. ${ }^{21}$

Composite reliability of the factors in the FF-NPQ was determined by evaluating Raykov's rho. ${ }^{18}$ Acceptable values of reliability range from 0.70 to $0.95 .{ }^{22}$ The reliability values of $0.70,0.80$ and 0.90 are considered adequate, very good and excellent, respectively. ${ }^{21}$

Test-retest reliability analysis was also performed to determine the consistency of reliability of the FFNPQ after one month using the intraclass correlation coefficient (ICC) for each factor. The ICC (two-way mixed, consistent type, single measures) was evaluated for this study purpose. ${ }^{23}$ The ICC value $<0.40$ is considered poor, that between 0.40 and 0.75 is considered fair to good and ICC > 0.75 is considered excellent. ${ }^{24}$

\section{Cutoff Values of the Scores}

The total scores of each factor obtained by the respondents were ranked in descending order. ${ }^{25}$ Then, the corresponding of the percentile to each observation was obtained. Three cutoff values were determined: 0th-24th percentile represents low, 25th-75th percentile represents moderate, 76th- 100th percentile represents high. ${ }^{26}$

\section{RESULTS}

Preliminary Data Screening and demographic profile A total of 155 observations was screened for any exclusion criteria met, missing values and wrong entry. Two observations were excluded from the analysis because of uncorrected visual acuity. Two observations were found to have missing values (1.3\%) that were considered low. However, these two observations were retained for further analysis, as MLR could handle both complete and incomplete data. ${ }^{27}$ Thus, 153 observations were used for further analysis. The majority of the respondents were female (85\%), Malay ethnicity (78\%) and were studying a bachelor degree (82\%). The mean age of all respondents was $22( \pm 2.13)$ years old.

\section{Internal Structure}

Out of the 153 observations, 151 complete ones were examined for multivariate normality. The data were not multivariate normal according to Mardia's test and chi-squared versus squared Mahalanobis distance (critical ratio of multivariate kurtosis = 11.89; $p$-value $<0.05$; points on the plot did not lie on a straight line).

In the CFA, the initial 60-item model (FF-NPQ model) did not fit the data well (Table I). Twentythree items had standardised factor loadings of less than 0.40 in the model (p11, p16, p21, p31 and p51 from Extraversion; p2, p12, p22, p32, p42, p52 and p57 from Agreeableness; p13, p23, p28 and p43 from Conscientiousness; p9, p19 and p59 from Neuroticism; and p15, p30, p54 and p60 from Openness to experience). The items were removed iteratively from the model, and 37 items remained. The CFA result indicated that an additional five items (p41 from Extraversion; p14, p29 and p39 from Neuroticism; and p50 from Openness to experience) from among the remaining items had low standardised factor loadings. The items were also removed from the model because they were socially unsuitable with the Malaysian culture and environment. Thirty-two items remained at this point. The 32-item model still did not achieve a satisfactory model fit. The MI values were reviewed to obtain a better model fit. High correlated errors between items in the same factor were considered for inclusion in the model. Correlations between factors were also reviewed to ensure no low factor 
loading (loading <0.40) and multicollinearity between factors $(r>0.85)$ could be found.

After the modifications, six items were removed from the model: three items ( $p 55$ from Neuroticism, p1 from Extraversion and p7 from Agreeableness) because of low factor loadings, two items (p49 from Neuroticism and p25 from Openness to experience) because of cross-loadings across factors and one item (p48 from Conscientiousness) because of a high standardised residual value of 3.29. Twenty-six items remained in this model (FF-NPQ revised model) with three correlated errors, as suggested by MI (Tables I and II). Thus, good fit indices (Table I), acceptable factor loadings (Table II) and no multicollinearity between factors (Table III) were obtained. The decreasing value of AIC and BIC indicated model improvement (Table I). Each factor had a good reliability, with all reliability values exceeding or close to 0.70 (Table II). Openness to Experience had the highest reliability, whereas Neuroticism had the lowest.

Table I: Comparison of goodness-of-fit indices between FF-NPQ model and FF-NPQ revised model

\begin{tabular}{llllllll}
\hline Model & No. of item & CFI & TLI & $\begin{array}{l}\text { RMSEA } \\
(90 \% \mathrm{CI})\end{array}$ & SRMR & AIC & BIC \\
\hline FF-NPQ & 60 & 0.485 & 0.464 & 0.069 & 0.102 & 32208.97 & 32782.25 \\
& & & $(0.065,0.073)$ & & & 13824.13 \\
\hline $\begin{array}{l}\text { FF-NPQ } \\
\text { revised }\end{array}$ & 26 & 0.958 & 0.952 & 0.026 & 0.068 & 14099.30 \\
\hline
\end{tabular}

Note: CFI = Comparative Fit Index; TLI = Tucker-Lewis Index; RMSEA = Root Mean

Square Error of Approximation; SRMR = Standardized Root Mean Square Residual;

$\mathrm{AIC}=$ Akaike Information Criteria; BIC = Bayesian Information Criterion

Table II: Factor loading, reliability and correlated errors in FF-NPQ revised model

\begin{tabular}{|c|c|c|c|c|c|}
\hline Factor & Item & $\begin{array}{l}\text { Factor } \\
\text { loading }\end{array}$ & Raykov's rho & Correlated errors & Correlation \\
\hline \multirow[t]{5}{*}{ Extraversion } & p6 & 0.456 & \multirow{5}{*}{0.72} & & \\
\hline & p26 & 0.624 & & & \\
\hline & p36 & 0.651 & & & \\
\hline & p46 & 0.670 & & & \\
\hline & p56 & 0.503 & & & \\
\hline \multirow[t]{4}{*}{ Agreeableness } & $\mathrm{p} 17$ & 0.571 & \multirow{4}{*}{0.69} & & \\
\hline & p27 & 0.715 & & & \\
\hline & p37 & 0.524 & & & \\
\hline & p47 & 0.602 & & & \\
\hline \multirow[t]{7}{*}{ Conscientiousness } & p3 & 0.454 & \multirow{7}{*}{0.76} & & \\
\hline & p8 & 0.463 & & & \\
\hline & $\mathrm{p} 18$ & 0.609 & & p33 and p58 & 0.297 \\
\hline & p33 & 0.468 & & p18 and p53 & -0.303 \\
\hline & p38 & 0.508 & & p3 and p18 & -0.237 \\
\hline & p53 & 0.624 & & & \\
\hline & p58 & 0.685 & & & \\
\hline \multirow[t]{4}{*}{ Neuroticism } & $\mathrm{p} 4$ & 0.643 & \multirow{4}{*}{0.68} & & \\
\hline & p24 & 0.709 & & & \\
\hline & p34 & 0.420 & & & \\
\hline & p44 & 0.540 & & & \\
\hline \multirow[t]{6}{*}{ Openness to experience } & p5 & 0.591 & \multirow{6}{*}{0.77} & & \\
\hline & $\mathrm{p} 10$ & 0.578 & & & \\
\hline & p20 & 0.685 & & & \\
\hline & p35 & 0.606 & & & \\
\hline & p40 & 0.501 & & & \\
\hline & p45 & 0.651 & & & \\
\hline
\end{tabular}

Note: $p=$ picture number 
Table III: Correlation between factors in FF-NPQ revised model

\begin{tabular}{lll}
\hline Factor & Factor & Correlation \\
\hline Extraversion & Agreeableness & 0.196 \\
& Conscientiousness & -0.013 \\
& Neuroticism & 0.136 \\
& $\begin{array}{l}\text { Openness to } \\
\text { Experience }\end{array}$ & 0.259 \\
\hline Agreeableness & Conscientiousness & 0.710 \\
& Neuroticism & 0.309 \\
& Openness to & 0.577 \\
\hline Conscientiousness & Neuroticism & 0.365 \\
& Openness to & 0.543 \\
& Experience & \\
\hline Neuroticism & Openness to & 0.051 \\
& Experience & \\
\hline
\end{tabular}

Test-retest reliability involved 44 participants randomly selected from the 153 participants in the initial data collection. Two observations were found to meet the exclusion criteria and excluded from the study, leaving 42 observations for further analysis. The value of Intraclass Correlation Coefficient (ICC) obtained for each personality factor ranged from 0.65 to 0.75 , which indicated fair to good reliability, as follows-Extraversion (0.67), Agreeableness (0.69), Conscientiousness (0.75), Neuroticism (0.75) and Openness to experience $(0.65)$.

\section{Cutoff Values of the Scores}

The cutoff values of the score for each factor were obtained by dividing them into three categories: low, moderate and high, as follows - Extraversion [five items: low (1-9), moderate (10-16), high (1735)], Agreeableness [four items: low (1-18), moderate (19-24), high (25-28)], Conscientiousness [seven items: low (1-35), moderate (36-44), high (45 -49)], Neuroticism [four items: low (1-14), moderate (15-21), high (22-28)] and Openness to experience [six items: low (1-25), moderate (26-34), high (3542)].

\section{DISCUSSION}

The FF-NPQ revised model fit the data well after removing 34 out of 60 pictorial items (56.7 per cent). The five-factor model was kept as it included all five dimensions of personality, namely Extraversion, Agreeableness, Conscientiousness,
Neuroticism and Openness to Experience. The finding was consistent with local studies on different personality inventory that used the same Big Five model tested on a sample of medical students in $U_{S M}{ }^{18,28}$ and on medical degree program applicants in USM, using the USM Personality Inventory (USMaPi). These two studies also reported five factors at the end of the study.

There were 26 items remained in the final model. Among the items removed were some with inappropriate illustrations that were not suitable with students' behaviour and Malaysian culture. For instance, item number 21 from Extraversion factor was removed due to low factor loading. The illustration shows that a person greets a new neighbour, where the situation is very common in Malaysia culture. Thus, the item did not contribute to Extraversion factor, because whether a person is high or low in extraversion, most Malaysians will practice this behaviour whenever they moved to a new place.

During assumption checking for CFA, the data were not multivariate normal. There were also missing values detected during data exploration and cleaning stage. In order to overcome the violation of multivariate normality, MLR was used when fitting the CFA model, which can be used in the existence of missing values. ${ }^{29}$ It can be applied on both complete and incomplete data. ${ }^{27}$ The MLR estimator is the estimation with robust (Huber-White) standard errors and a scaled test statistic that is asymptotically equal to the Yuan-Bentler test statistic. $^{27}$

The factors of FF-NPQ had good reliability as indicated by the reliability coefficients close or exceeding the 0.70 , which contradicted the study by Nur Farliza et al. ${ }^{28}$ where the reliability was poor. However, the reliability in this study was consistent with the study implemented in Czech on psychometric properties of $\mathrm{FF}-\mathrm{NPQ}^{30}$, which resulted in four scales had good reliability of higher than 0.70 and only Conscientiousness scale had reliability below than 0.70 .

From the test-retest reliability analysis on 42 respondents after one month duration, the ICC obtained for each personality ranged from fair to good reliability. 


\section{CONCLUSION}

The revised Malay Version of FF-NPQ showed good validity and maintained the broad five-factor personality dimensions. A cross-validation study is recommended to replicate the findings of the present study in view of the reduced number of items in the revised FF-NPQ.

\section{ACKNOWLEDGEMENT}

The authors are grateful to Universiti Sains Malaysia (USM), Kubang Kerian, Kelantan, Malaysia for the financial support under the USM Research University Grant (Grant Number: 1001/PPSP/812188) as well as research facilities provided.

\section{DECLARATION OF CONFLICTING INTERESTS}

The authors declare no potential conflicts of interest with respect to the research, authorship and publication of this article.

\section{REFERENCES}

1. Roberts BW, Mroczek D. Personality trait change in adulthood. Curr Dir Psychol Sci. 2008; 17(1): 31-35.

2. Schultz DP, Schultz SE. Theories of personality. Belmont, CA: Wadsworth Cengage Learning; 2009.

3. Bhagat V. Extroversion and academic performance of medical students. IJHSSI. 2013; 2(3): 55-58.

4. John OP, Srivastava S. The Big Five trait taxonomy: History, measurement, and theoretical perspectives. In: Handbook of personality: Theory and research (Vol. 2, pp. 102-138). New York: Guilford; 1999.

5. Gosling SD, Rentfrow PJ, Swann WB. A very brief measure of the Big-Five personality domains. J Res Pers. 2003; 37(6): 504-528.

6. Paunonen SV, Jackson DN, Ashton MC. NPQ Manual: Nonverbal Personality Questionnaire (NPQ) and Five-Factor Nonverbal Personality Questionnaire (FF-NPQ). New York: Sigma Assessment Systems; 2004.

7. Paunonen SV, Ashton MC, Jackson DN. Nonverbal assessment of the big five personality factors. Eu J Pers. 2001; 15(1): 318.

8. Preacher KJ, Coffman DL. Computing power and minimum sample size for RMSEA; 2006.

9. Brown TA. Confirmatory Factor Analysis for Applied Research: The Guilford

Press; 2006.

10. Hair J, Black WC, Babin BJ, Anderson RE. Multivariate Data Analysis. 7th ed. Pearson Prentice Hall; 2010.

11. Beaton DE, Bombardier C, Guillemin F, Ferraz $M B$. Guidelines for the process of cross-cultural adaptation of self-report measures. Spine. 2000; 25(24): 3186-3191.

12. Guillemin F, Bombardier C, Beaton D. Crosscultural adaptation of health-related quality of life measures: Literature review and proposed guidelines. J Clin Epidemiol. 1993; 46(12): 1417 $-1432$.

13. Korkmaz S, Goksuluk D, Zararsiz G. MVN: an R package for assessing multivariate normality. $R$ J. 2014;6(2):151-62.

14. Nimon KF. Statistical Assumptions of Substantive Analyses Across the General Linear Model: A Mini-Review. Front Psychol [Internet]. 2012;3:322. Available at: http:// journal.frontiersin.org/article/10.3389/ fpsyg.2012.00322/abstract. Accessed February 21, 2018.

15. Burdenski T. Evaluating Univariate, Bivariate, and Multivariate Normality Using Graphical Procedures. Mult Linear Regres Viewpoints. 2000;26(2):15-28.

16. Rios J, Wells C. Validity evidence based on internal structure. Psicothema. 2014;26(1):108 16.

17. Brown TA. Confirmatory factor analysis for applied researchers. New York: Guilford Press; 2007.

18. Hu L, Bentler PM. Cutoff criteria for fit indexes in covariance structure analysis: Conventional criteria versus new alternatives. Struct Equ Model A Multidiscip J. 1999;6(1):1-55.

19. DeVon $\mathrm{H}$ a., Block ME, Moyle-Wright $\mathrm{P}$, et al. A psychometric toolbox for testing validity and reliability. J Nurs Scholarsh. 2007;39(2):155-64.

20. Hair JF, Black WC, Babin BJ, Anderson RE. Multivariate Data Analysis. 7th ed. Upper Saddle River, New Jersey: Pearson Prentice Hall; 2010.

21. Kline RB. Principles and Practice of Structural Equation Modeling. 3rd ed. New York: The Guildford Press; 2011.

22. Tavakol M, Dennick R. Making sense of Cronbach's alpha. Int J Med Educ [Internet]. 
2011;2:53-5. Available at: http://

www.ijme.net/archive/2/cronbachs-alpha/. Accessed from February 20, 2018.

23. Trevethan R. Intraclass correlation coefficients: clearing the air, extending some cautions, and making some requests. Heal Serv Outcomes Res Methodol [Internet]. 2016;1-17. Available at: http:// link.springer.com/10.1007/s10742-016-0156-6. Accessed from February 20,2018.

24. Fleiss JL. The Design and Analysis of Clinical Experiments. Canada: John Wiley \& Son, Inc; 1986.

25. Streiner DL, Norman GR, Cairney J. Health measurement scales: a practical guide to their development and use. 5th ed. New York: Oxford University Press, USA; 2015.

26. Yusoff MSB. Construct validity, internal consistency and normative data of the USMaP-i in a sample of medical students. Int Med J. 2013;20(1):1-7.

27. Beaujean AA. Latent variable modeling using R: A step-by-step guide. New York and East Sussex: Routledge; 2014.

28. Nur Farliza S, Arifin WN, Yusoff MSB, Yaacob NA, Naing NN. A Confirmatory Factor Analysis of USM Personality Inventory (USMaP-i) among Medical Degree Program Applicants in Universiti Sains Malaysia. Educ Med J. 2016;8 (1):55-65.

29. Raykov T, Marcoulides GA. Scale Reliability Evaluation Under Multiple Assumption Violations. Struct Equ Model A Multidiscip J [Internet]. 2016;23(2):302-13. Available at: http://www.tandfonline.com/doi/ full/10.1080/10705511.2014.938597. Accessed from February 20, 2018.

30. Hrebickova M. The Five-Factor Nonverbal Personality Questionnaire in The Czech Context. Stud Psychol (Bratisl). 2010;52(3):165 -77. 